\title{
Spatial solitons in a pumped semiconductor resonator
}

\author{
V. B. Taranenko, C. O. Weiss, W. Stolz* \\ Physikalisch-Technische Bundesanstalt 38116 Braunschweig, Germany \\ * Philipps Universitaet, 35032 Marburg, Germany
}

\begin{abstract}
Bright and dark spatial solitons are observed in an optically pumped semiconductor resonator. The pumping allows to considerably reduce the light intensity necessary for the existence of the solitons and alleviates thermal load problems. Experiments are found to agree with calculations based on a simple large aperture semiconductor resonator model. The role of signs of the absorptive and reactive nonlinearities for soliton existence are discussed in relation with the nonlinear resonance effect, the tilted wave mechanism of pattern formation, and the sign of the population inversion.
\end{abstract}

PACS 42.65.Sf, 42.65.Pc, 47.54.+r

Spatial resonator solitons are presently considered for optical information processing, in the form of mobile binary information carriers [1]. We have recently shown that such solitons exist in semiconductor quantum well resonators [2]. The speed with which these solitons can be switched-on was, however, found to be limited to $\sim 1$ $\mu \mathrm{s}$, as a consequence of the high local heat dissipation inside the bright solitons [2, 3]. These thermal effects limit also the speed with which these solitons can be moved. The high dissipation in the solitons relates directly to the high light intensity at which these solitons exist. Typical intensities supporting the solitons are $\sim 1 \mathrm{~kW} / \mathrm{cm}^{2}$ or the power needed to support one soliton of $\sim 10 \mu \mathrm{m}$ size is $\sim 1 \mathrm{~mW}$; a sizeable fraction of which is dissipated.

To reduce the high light intensities needed to support solitons, and with this to ease the limitations due to dissipation, we attempt to use a pumped medium rather than a purely absorbing one inside the resonator. Conceptually the idea being that part of the energy supporting the solitons be provided incoherently, reducing the energy to be supplied in a coherent form. For simplicity and flexibility we have used optical pumping in the experiment, which could, of course, be replaced by electrical pumping in the technical applications.

The set-up for the observations is largely as described in [2]. In short, it uses a $c w$ Ti: $\mathrm{Al}_{2} \mathrm{O}_{3}$-laser tunable to chose the desired wavelength region - in our case near the semiconductor band edge. This laser illuminates an area of $\sim 50 \mu \mathrm{m}$ diameter on the semiconductor resonator sample, corresponding to a Fresnel number of $\sim 100$. The light reflected from the sample is observed, taking nssnapshots of the illuminated area and following the reflected intensity in time in particular points in the area. Details are given in [2, 3].

For optical pumping light from a $750 \mathrm{~mW}$ diode laser with spectral width $\sim 3 \mathrm{~nm}$, and thus rather incoherent, is spatially superimposed onto the $\mathrm{Ti}: \mathrm{Al}_{2} \mathrm{O}_{3}$-laser light. The resonator sample used, described in [4], contains 18 quantum wells and has mirrors of $\sim 99.6 \%$ reflectivity. The optical pumping wavelength was chosen as $810 \mathrm{~nm}$ (about $40 \mathrm{~nm}$ shorter than the band gap wavelength) in the stop band of the resonator mirrors 四. The coherent laser radiation near the band edge wavelength was, as usual for soliton formation, blue-detuned with respect to the resonator resonance (tilted wave mechanism for structure formation, see [5]).

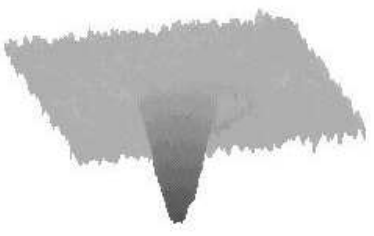

a)

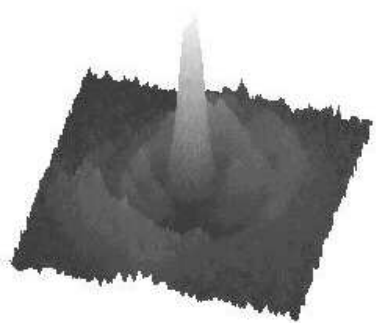

b)
FIG. 1. Spatial resonator solitons in the pumped semiconductor QW-resonator. Observation in reflection. a) bright soliton (view from "bottom"), b) dark soliton (normal or "top"-view). The vertical coordinate is the sample reflectivity. Pump strength is near the crossing point of the switching thresholds in Fig. 2.

We find that bright and dark spatial resonator solitons exist throughout the whole range of available pump intensities (see Fig. 1). Fig. 2 shows the variation of the switch-on and switch-off intensities of the bistable resonator, with the pump intensity. It is seen that the light intensities supporting spatial solitons are reduced at the maximum of the pump power available here by nearly an order of magnitude, compared to the unpumped case. This should constitute a significant advantage in applications. The measurements were routinely carried out illuminating the resonator sample for a few ms only, to limit thermal phenomena. The switching thresholds in Fig. 2a were determined from recordings such as Fig. 4a where the switch-on and -off intensities are evident. For higher pump intensities Fig. 2a shows that the switch-on intensities, measured in this way, lie below the switch-off intensities. We would interpret this as an artifact due to 
a residual thermal effect: At the time of switch-on, the material is "cold". When the material is switched on, the intensity inside the soliton and with it the dissipation is high, thus at the time of switch-off the material temperature is increased, and the band gap, and with it the bistability characteristic, has shifted. The switch-off intensity is then increased over the value of the "cold" material and thus the measured switch-on and switch-off thresholds appear to cross.
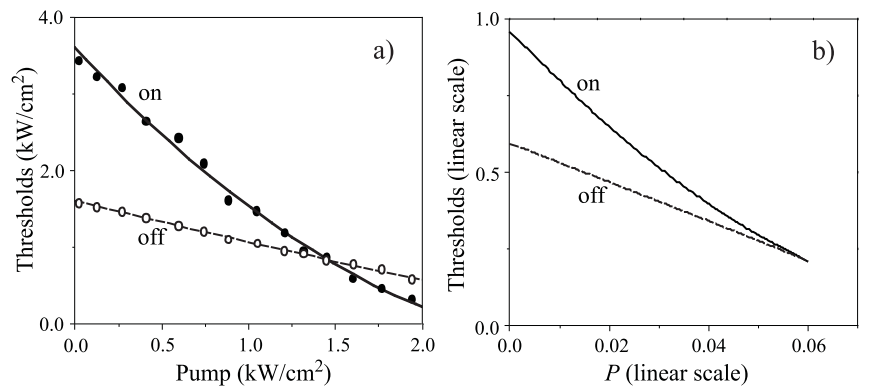

FIG. 2. a) Measured switch-on and switch-off intensities of the bistable resonator as a function of pump strength. Solid and dashed lines given to guide the eye. b) Calculated plane wave switching intensities obtained from model (1). Parameters are $\eta=0.5, C=36, \operatorname{Im}(\alpha)=1, \operatorname{Re}(\alpha)=-0.02, \theta=-2$, $\gamma=0.1$. Transparency corresponds to $P=0.1$.

In the presence of pumping the power dissipated locally (in the soliton) which causes the limitations in switching and movement speed, mentioned above, is reduced according to the switching threshold. This can be expected to alleviate the limitations given by dissipation significantly.

Fig. $2 \mathrm{~b}$ shows the switching thresholds for plane waves as calculated from the rate equation model for the intracavity optical field $E$ and carrier density $N$ (similar to [6]):

$$
\left\{\begin{array}{r}
\partial E / \partial t=E_{\text {in }}-E[1+\eta+C \operatorname{Im}(\alpha)(1-N)]- \\
-i E\left[\theta-C \operatorname{Re}(\alpha) N-\nabla_{\perp}^{2}\right] \\
\partial N / \partial t=P-\gamma\left[N-|E|^{2}(1-N)-d \nabla_{\perp}^{2} N\right]
\end{array}\right.
$$

where $E_{\text {in }}$ is the incident field, $\eta$ is the linear intracavity absorption, $C$ is the bistability parameter [6], $\operatorname{Im}(\alpha)(1-$ $N)$ and $\operatorname{Re}(\alpha) N$ are used to describe the absorptive and refractive nonlinearities, respectively. $\theta$ is the detuning of the optical field from the resonator resonance, $\gamma$ is the ratio of the photon lifetime in the resonator to the carrier nonradiative recombination time. $P$ is the optical pump rate, $d$ is the diffusion coefficient (normalized to the diffraction coefficient) and $\nabla_{\perp}^{2}=\partial^{2} / \partial x^{2}+\partial^{2} / \partial y^{2}(x, y$ are the transverse coordinates) accounts for nonlocalities. Figs $2 \mathrm{a}$ and $2 \mathrm{~b}$ show a good qualitative agreement.

Fig. 3 shows actual numeric soliton solutions of (1) as they are found for pump values close to the point where the on- and off-thresholds for plane waves (Fig. 2b) intersect (small hysteresis range). The steady state, plane wave characteristic (in reflection) of the resonator is given. For the same value of resonator detuning bright solitons appear closely below the plane wave switch-on and dark solitons closely above it.

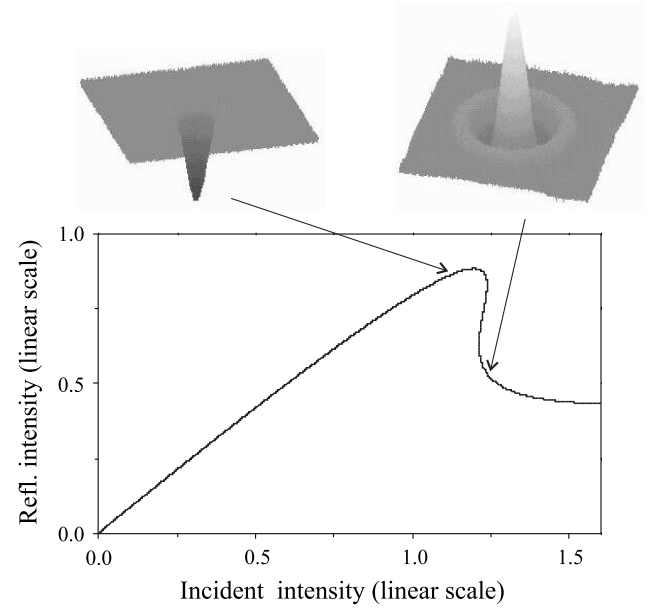

FIG. 3. Plane wave resonator characteristics calculated from model (1). Bright and dark soliton solutions shown on top exist close to the bistability range. Parameters are $\eta=0.5, C=36, \operatorname{Im}(\alpha)=1, \operatorname{Re}(\alpha)=-0.02, \theta=-2$, $P=0.055, \gamma=0.1, d=0.1$.

Fig. 4 shows observation of the dynamic behavior of the bright and dark soliton as measured under these conditions. Fig. 4a is the case of bright solitons. The intensity reflected from the center of the soliton is given. Without pumping the resonator does not switch and reflects all incident light. For the same illuminating intensity the soliton switches on with pumping. The switch-on is apparently direct and fast and not mediated by thermal effects as for soliton formation without pumping [2].

Fig. 4b shows the light reflected for a dark soliton. Without pumping the sample switches an area given by the illuminating beam at $\sim 0.4 \mu \mathrm{s}$. No soliton forms here. With pumping the switching appears at a much lower intensity and a dark soliton is formed because the illuminating intensity is higher than in Fig. 4a. The slow change of intensity measured over the time of observation reflects a motion of the dark soliton, as has been shown in [2]. This motion is a consequence of the temperature difference between the interior and the exterior of the soliton. Such temperature difference does not allow a dark soliton to sit stationarily in one location, but forces it to move continually [7], much in the way discussed for optical vortices in class B-lasers [8]. We note here (as in [2]) that the reflectivity at the soliton center is larger than 1 (!), indicating that the soliton collects energy from its surrounding.

In [2, 3] we found that under normal conditions the 
formation of bright solitons is slowed by a process determined by the heating due to the high intensity inside the soliton. We note here that a time-(and space-) independent heating does not produce such problems. This is evidenced by the direct switch-on of the soliton in Fig. 4a. The uniform heating due to the pump causes only a timeindependent shift of the bistability characteristics (together with the basin of attraction for solitons), altering switching thresholds, but nothing else. Thus the heating by the pump does not slow soliton formation. Rather it allows the reduction of the soliton supporting coherent light intensity, and with it the time- and space-dependent heating.

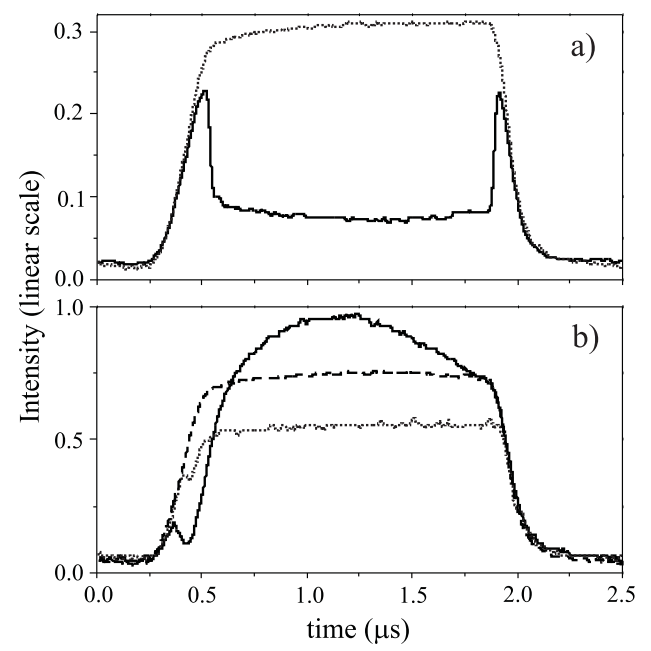

FIG. 4. Dynamical features of bright soliton (a)) and dark soliton (b)).

a) Dotted line: reflected intensity without pumping. The switch-on intensity is not reached, thus incident intensity equals the reflected intensity. Solid line: with pumping, a soliton switches on directly (without delay from thermal effects) at $0.5 \mu \mathrm{s}$, and off at a slightly smaller intensity. Pump strength near the threshold crossing point of Fig. 2 (as in Fig. 1).

b) Dashed line: incident intensity. Dotted line: reflected intensity without pumping. A certain area in the illumination beam is switched on at $0.5 \mu \mathrm{s}$, but no soliton formed. Solid line: reflected intensity with pumping. A dark soliton is switched on at $0.5 \mu \mathrm{s}$. Slow changes of the reflected intensity are due to motion of the soliton (see text).

Intensity scales of a), b) normalized to the same value.

The pumping allows i) to reduce dramatically the light intensity supporting the solitons, ii) to switch on solitons fast without thermal delay. This would also imply that with pumping thermal effects would not limit the speed with which solitons can be moved.

In these pumping experiments we have worked below population inversion in the quantum-well-material. This is concluded in the following way: At the transparency point all material nonlinearities can be expected to vanish and with them any bistability. We have not crossed that point with the pump intensities available to us (Fig. 2) as bistability is found up to the highest pump value.

For our sample with its high reflectivity mirrors one would also think that the transparency point and the laser threshold would be extremely close in pump intensity. The resonator needs only inversion of $1 \%$ above transparency to emit as a laser, so that it would be difficult to keep the pump power in this very narrow range.

We mention one essential difference in bistability and solitons below and above transparency. Below transparency the absorptive nonlinearity (bleaching) has the correct sign for a resonator bistability, while above transparency (gain saturation) the sign is wrong. Consequently, below transparency solitons based on the dissipative part of the nonlinearity can exist, while above such solitons can not exist. Evidently, below, as well as above transparency, solitons can be supported by the reactive (dispersive) part of the nonlinearity.

Additionally concerning the reactive part of the nonlinearity, there is also a difference below and above transparency. The nonlinear resonance mechanism of soliton formation [9] requires (counter intuitively) a defocusing nonlinearity below transparency and a focusing nonlinearity above transparency for a blue-detuned resonator. Self-focusing is generally furthering soliton formation. Working above transparency, would therefore seem to be advantageous for solitons.

In summary, we have shown the beneficial effects of pumping on the properties of spatial resonator solitons. The necessary light intensities can be drastically reduced and thermal problems hindering fast switching and fast motion are reduced. How close one can work above or below the transparency point, i.e. how small the coherent intensities supporting solitons can be, depends evidently on the requirements of soliton stability, as the existence ranges reduce, as one approaches transparency. Although we have used optical pumping for convenience here, the option exists to replace it by electrical pumping in applications.

Acknowledgement

We acknowledge beneficial discussions with D.Michaelis and K.Staliunas. Work supported by ESPRIT under project number 28235 and Deutsche Forschungsgemeinschaft under project number we743/12-1.

[1] See e.g. W. J. Firth, G. K. Harkness, Asian J. Phys. 7, 665 (1998).

[2] V. B. Taranenko, I. Ganne, R. Kuszelewicz, C. O. Weiss, Appl. Phys. B 72, 377 (2001).

[3] V. B. Taranenko, C. O. Weiss, Appl.Phys B 72, (2001) in press. 
[4] B. G .Sfez, J. L. Oudar, J. C. Michel, R. Kuszelewicz, R. Azoulay, Appl.Phys.Lett. 57, 1849 (1990); I. Abram, S. Iung, R. Kuszelewicz, G. LeRoux, C. Licoppe, J. L. Oudar, Appl.Phys.Lett. 65, 2516 (1994).

[5] P. K. Jakobsen, J. V. Moloney, A. C. Newell, R. Indik, Phys.Rev. A 45, 8129 (1992).

[6] L. Spinelli, G. Tissoni, M. Brambilla, F. Prati, L.A. Lugiato, Phys.Rev. A 58, 2542 (1998).

[7] L. A. Lugiato, private communication.

[8] C. O. Weiss, H. R. Telle, K. Staliunas, M. Brambilla, Phys.Rev. A 47, R1616 (1993).

[9] G. J. de Valcarcel, K. Staliunas, V. J. Sanchez-Morcillo, E. Roldan, Phys. Rev. A 54, 1609 (1996). 